\title{
GMR
}

\section{CADM1 mRNA expression and clinicopathological significance in esophageal squamous cell carcinoma tissue}

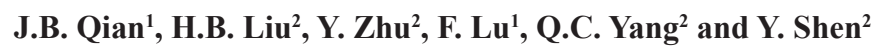 \\ ${ }^{1}$ Department of Digestive Diseases, Nantong First People's Hospital, \\ Nantong, Jiangsu, China \\ ${ }^{2}$ Department of Pathology, Nantong First People's Hospital, Nantong, \\ Jiangsu, China \\ Corresponding author: H.B. Liu \\ E-mail: liuhongbin_hb@163.com \\ Genet. Mol. Res. 16 (2): gmr16029178 \\ Received September 6, 2016 \\ Accepted April 24, 2017 \\ Published May 10, 2017 \\ DOI http://dx.doi.org/10.4238/gmr16029178
}

Copyright $(92017$ The Authors. This is an open-access article distributed under the terms of the Creative Commons Attribution ShareAlike (CC BY-SA) 4.0 License.

ABSTRACT. The mRNA expression of cell adhesion molecule 1
(CADM1) and its clinicopathological significance in esophageal
squamous cell carcinoma (ESCC) tissues were investigated. CADM1
mRNA and protein expression were detected in tissue samples from 50
patients with ESCC by reverse transcription-polymerase chain reaction
(RT-PCR) and streptavidin-peroxidase (SP) immunohistochemistry;
adjacent tissues served as controls. The average CADM1 mRNA
expression was significantly downregulated in the cancer tissues $(0.522$
$\pm 0.247)$ than in the controls $(0.871 \pm 0.192),(t=7.882, \mathrm{P}<0.05)$.
CADM1 mRNA expression was significantly downregulated in ESCC
patients with positive lymph node metastasis than in those with negative
lymph node metastasis $(t=3.207, \mathrm{P}<0.05)$. There was a correlation
between CADM1 mRNA expression and tumor-node-metastasis
(TNM) stage $(t=2.673, \mathrm{P}<0.050)$, but not with age, gender, and
histological grade $(\mathrm{P}>0.05)$. The positive expression rate of CADM1

Genetics and Molecular Research 16 (2): gmr16029178 
protein in the 50 cases of ESCC was significantly lower than that of the control group $\left(\chi^{2}=29.87, \mathrm{P}<0.01\right)$. Out of 28 patients with nonlymph node metastasis, 20 (71.43\%) positively expressed CADM1; out of 22 patients with lymph node metastasis, only 7 (31.82\%) positively expressed CADM1. There was a significant difference in the positive protein expression rates of CADM1 between the two groups $\left(\chi^{2}=\right.$ 7.782, $\mathrm{P}<0.01)$. CADM1 mRNA expression was highly upregulated in normal tissues compared to ESCC tissues, indicating that the loss of CADM1 expression influenced the pathogenesis, invasion, and metastasis of ESCC, and allowing for the prognosis of the disease in patients with ESCC after treatment.

Key words: Esophageal squamous cell carcinoma; CADM1; mRNA; RT-PCR; SP immunohistochemical method

\section{INTRODUCTION}

Esophageal squamous cell carcinoma is one of the most malignant types of cancer with the sixth highest global-mortality rate. China has high incidence and mortality rates of ESCC. Although there have been some improvements in the diagnosis and treatment of ESCC, the incidence and mortality rate are still high. According to statistics, the survival rate in 5 years is $10 \sim 15 \%$ (Kuramochi et al., 2001). The inhibition of lymph node metastasis and invasion of ESCC is critical to treatment and improvements in survival rate.

Cell adhesion molecule 1 (CADM1) was identified by useful complementation method as a tumor-inhibiting factor (TIF), while researching lung cancer (Kuramochi et al. 2001). CADM1 expression was downregulated and heterozygosity was lost in $85 \%$ of the primary tumors (Liang et al., 2011).

In this research study, we used reverse-transcription polymerase chain reaction (RT-PCR) and streptavidin-peroxidase (SP) immunohistochemistry to detect mRNA and protein expression levels of CADM1 in ESCC tissues and investigate its clinicopathological significance.

\section{MATERIAL AND METHODS}

Informed consent was obtained from all the patients and the study was approved by the Ethics Committee of the Department of Pathology, Nantong People's Hospital. Data and ESCC tissue samples were collected from 50 patients admitted to the Nantong People's Hospital from January 2010 to December 2014. All the patients were diagnosed with ESCC by using histopathology and no patient received radiotherapy or chemotherapy before the operation. Becrosis-free tissues were freshly collected, and then stored in liquid nitrogen at $-80^{\circ} \mathrm{C}$. There were 35 males and 15 females within an age range of $45-79$ years (with a mean of 62 years). According to the international tumor-node-metastasis (TNM) staging system, there were 7 cases in stage I, 20 cases in stage II, 18 cases in stage III, and 5 cases in stage IV.

\section{RNA extraction}

Fifty milligrams of tissue was cut into cubes and homogenized at $4^{\circ} \mathrm{C}$. Total RNA was

Genetics and Molecular Research 16 (2): gmr16029178 
extracted using TRIzol reagent (Beyozol Co., USA). RNA samples $(10 \mu \mathrm{L})$ were loaded and analyzed using agarose gel electrophoresis to determine purity.

Based on the CADM1 mRNA sequence available in the GenBank database, CADM1 primers were designed with the help of Premier 5.0 software having a size of 440 base pairs. The sequence of the forward primer was 5'-GCAGGTGAAGAAGGCTCGAT-3' and that of the reverse primer was $5^{\prime}$-CCCAGAATGATGAGCAAGCA-3'. $\beta$-actin was taken as an internal parameter with the forward primer as 5'-TTCCAGCCTTCCTTCCTGG-3' and reverse primer as 5'-TTGCGCTCAGGAGGAGCAAT-3'. The primers were synthesized by Shanghai Sangon Biotech Co., Ltd. (China). The polymerase chain reaction (PCR) conditions were as follows: 40 cycles of pre-denaturation at $95^{\circ} \mathrm{C}$ for $1 \mathrm{~min}$, denaturation at $95^{\circ} \mathrm{C}$ for $10 \mathrm{~min}$, annealing at $95^{\circ} \mathrm{C}$ for $15 \mathrm{~s}$, and extension at $60^{\circ} \mathrm{C}$ for $1 \mathrm{~min}$.

\section{PCR result}

The PCR products were analyzed on 1.5\% agarose gel and scanned using Eagle Eye II image analysis system. The ratio of optical density of CADM1 to that of $\beta$-actin was used for representing the CADM1 mRNA expression.

\section{Detection of CADM1 proteins by SP immunohistochemical method}

All samples were sectioned into $4-\mu \mathrm{m}$ slices, fixed with $10 \%$ neutral formalin solution, and embedded in paraffin. The samples were stained using the hematoxylin and eosin (HE) and SP immunohistochemical stains. The primary anti-CADM1 antibody used was rabbit anti-human monoclonal antibody (1:100 dilutions), purchased from Beijing Zhongjin Jinqiao Biotechnology Co., Ltd. (China). It was graded according to the percentage of positive cells: 0 points: no positive cells, 1 point: percentage of positive cells $<30 \%, 2$ points: percentage of positive cells of the range $30-70 \%, 3$ points: percentage of positive cells $>70 \%$; and also according to the intensity of color: 0 points: no positive cells, 1 point: positive cells stained pale yellow, 2 points: positive cells stained yellow, 3 points: positive cells stained brown. The sum of the two scores was considered the final score: 0 points (-), 1-2 points (+), 3-4 points $(++)$, and 5-6 points $(+++) .(-)$ and $(+)$ were categorized into a negative group and $(++)$ and $(+++)$, a positive group.

\section{Statistical analysis}

All data were analyzed by using the Stata 7.0 software. The levels of CADM1 mRNA expression are reported as means $\pm \mathrm{SD}$. The $t$-test was used for comparisons between the different groups. A value of $\mathrm{P}<0.05$ was considered to be statistically significant.

\section{RESULTS}

\section{CADM1 mRNA expression in esophageal squamous cell carcinoma}

The levels of CADM1 mRNA expression were highly upregulated in ESCC tissues. The average CADM1 mRNA expression level in the control tissues from 50 cases was $0.871 \pm 0.192$, which was higher than that of the tumor tissues $(0.522 \pm 0.247)(t=7.882, \mathrm{P}<0.01)$ (Figure 1).

Genetics and Molecular Research 16 (2): gmr16029178 


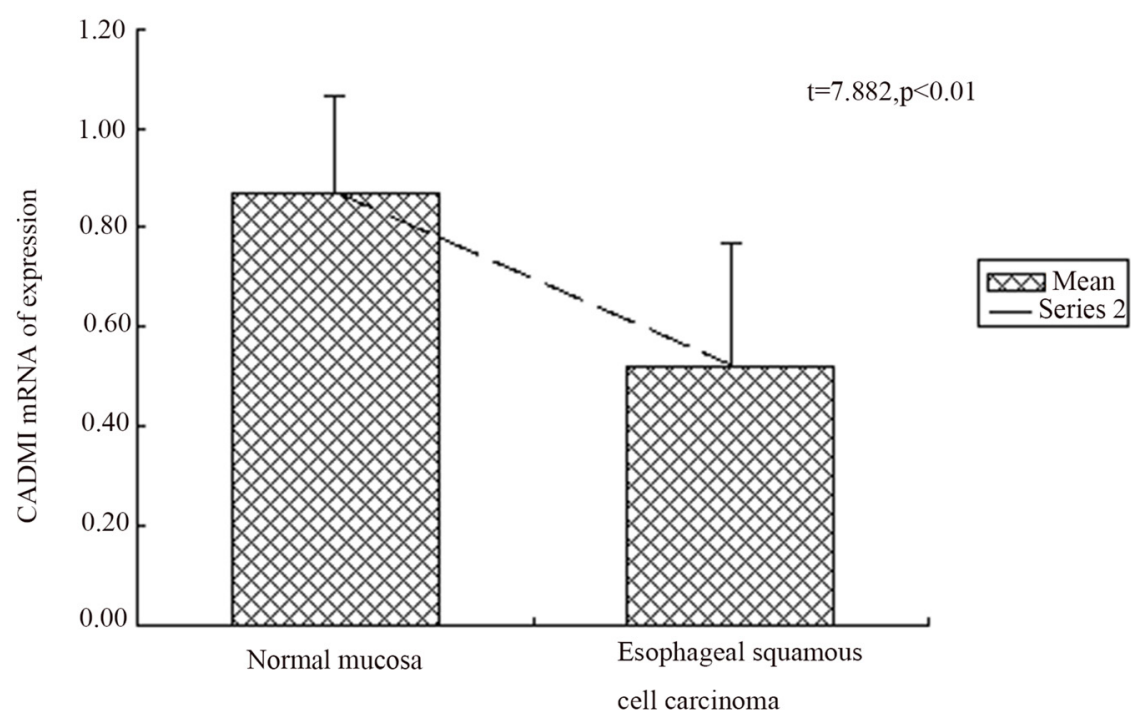

Figure 1. CADM1 mRNA expression in tumor and adjacent tissues of 50 patients with ESCC.

\section{CADM1 mRNA expression and clinicopathological factors}

In all the 50 cases of ESCC, CADM1 mRNA expression levels were downregulated with increase in histological grading, but there was no significant difference $(t=0.864, \mathrm{P}>$ 0.05 ) (Figure 2). CADM1 mRNA in those patients with lymph node metastasis was lower than that of those without lymph node metastasis $(t=3.027, \mathrm{P}<0.01)$ (Figure 3$)$. There was a significant difference in the CADM1 mRNA expression levels between stages I+II and stages III+IV $(t=2.673, \mathrm{P}<0.05)$ (Figure 4). There was no correlation between CADM1 mRNA expression and clinicopathological factors such as gender and age $(\mathrm{P}>0.05)$ (Table 1).

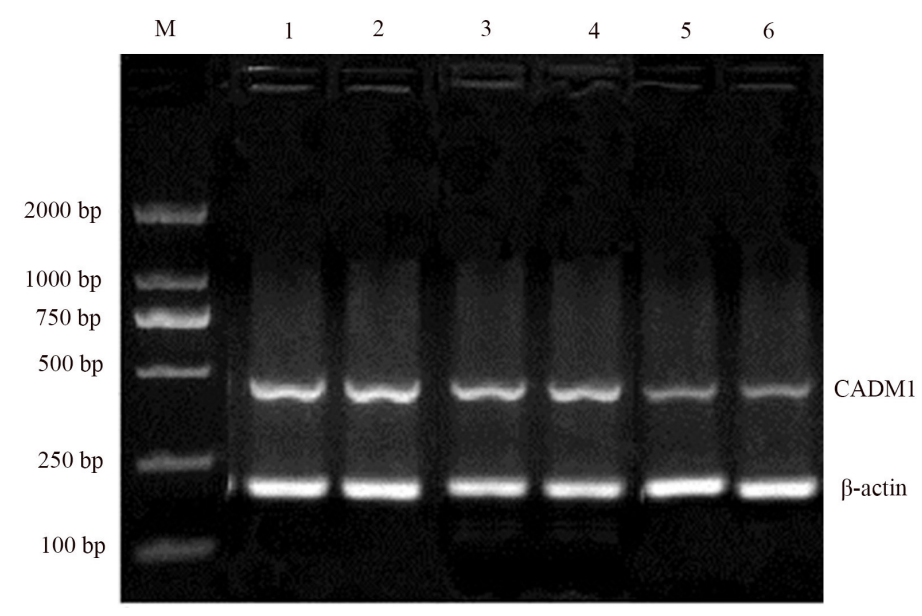

Figure 2. CADM1 mRNA RT-PCR amplification. Lane $M=$ DNA Marker; lanes 1 and $2=$ TNM grade I; lanes 3 and $4=$ TNM grade II; lanes 5 and $6=$ TNM grade III.

Genetics and Molecular Research 16 (2): gmr16029178 


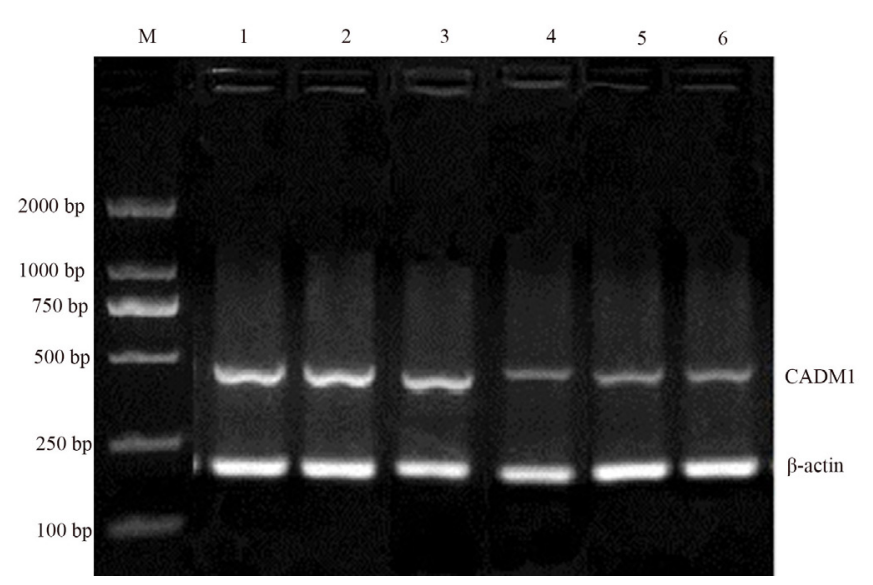

Figure 3. CADM1 mRNA RT-PCR amplification. Lane $M=$ DNA Marker; lanes 1,2 and $3=$ ESCC without lymph node metastasis; lanes 4, 5 and $6=\mathrm{ESCC}$ with lymph node metastasis.

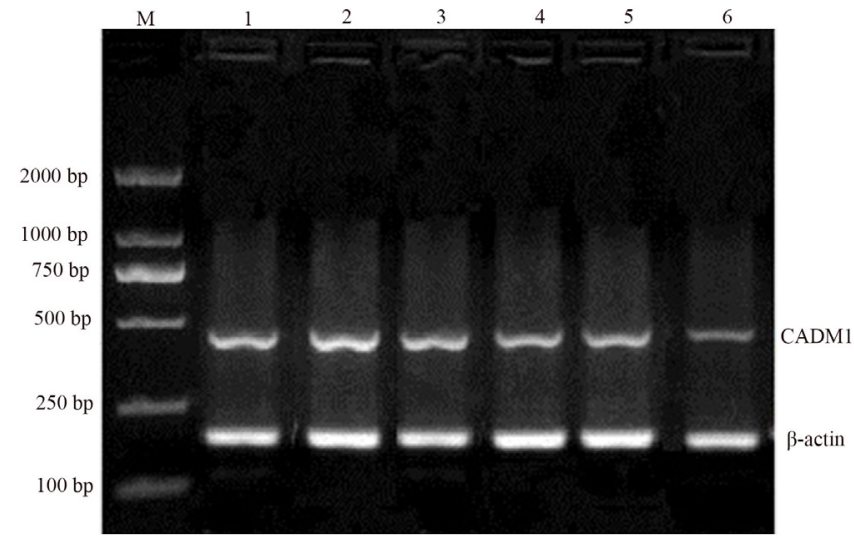

Figure 4. CADM1 mRNA RT-PCR amplification. Lane $M=$ DNA Marker; lane $1=$ TNM grade I; lanes 2 and $3=$ TNM grade II; lanes 4 and 5 ; TNM grade III; lane $6=$ TNM grade IV.

Table 1. Correlation of CADM1 mRNA expression with clinicopathological factors in 50 cases of ESCC.

\begin{tabular}{|c|c|c|c|c|}
\hline Groups & Cases & CADM1 mRNA (means $\pm \mathrm{s}$ ) & $t$ & $\mathrm{P}$ \\
\hline Gender & & & 0.143 & 0.887 \\
\hline Male & 35 & $0.527 \pm 0.312$ & & \\
\hline Female & 15 & $0.514 \pm 0.247$ & & \\
\hline Age (years) & & & -0.540 & 0.591 \\
\hline$\leq 60$ & 21 & $0.505 \pm 0.146$ & & \\
\hline$>60$ & 29 & $0.537 \pm 0.241$ & & \\
\hline TNM grade & & & 0.864 & 0.104 \\
\hline I & 12 & $0.724 \pm 0.271$ & & \\
\hline II & 23 & $0.521 \pm 0.178$ & & \\
\hline III & 15 & $0.413 \pm 0.264$ & & \\
\hline Lymph node metastasis & & & 3.207 & 0.002 \\
\hline Positive & 28 & $0.634 \pm 0.246$ & & \\
\hline Negative & 22 & $0.435 \pm 0.175$ & & \\
\hline TNM grade* & & & 2.673 & 0.010 \\
\hline $\mathrm{I}+\mathrm{II}$ & 27 & $0.612 \pm 0.219$ & & \\
\hline III+IV & 23 & $0.451 \pm 0.204$ & & \\
\hline
\end{tabular}

Data are reported as means \pm SD. ${ }^{*}$ Grades I and II have been combined and grades III and IV have been combined.

Genetics and Molecular Research 16 (2): gmr16029178 


\section{Detection of the expression of CADM1 protein in esophageal squamous cell carcinoma by immunohistochemical analysis}

The cells with positive expression of the CADM1 protein in normal esophageal tissues showed the presence of brown granules in the cytoplasm, and the cells with positive reaction were widely distributed throughout the esophageal tissue including the whole layer of the esophageal epithelium (Figure 5); 50 patients with normal esophageal tissues exhibited a strong positive reaction, and the expression rate of CADM1 protein was $100.0 \%$. The positively expressed CADM1 protein in ESCC tissues appeared as light yellow, yellow, or brownish granules in the cytoplasm (Figures 6-8). There were 27 out of 50 cases of esophageal cancer with positive expression, and the positive expression rate of CADM1 protein was 54\%. There was a statistically significant difference in the positive expression rate of CADM1 protein between esophageal carcinoma tissues and normal esophageal tissues $\left(\chi^{2}=29.87, \mathrm{P}<0.01\right)$.

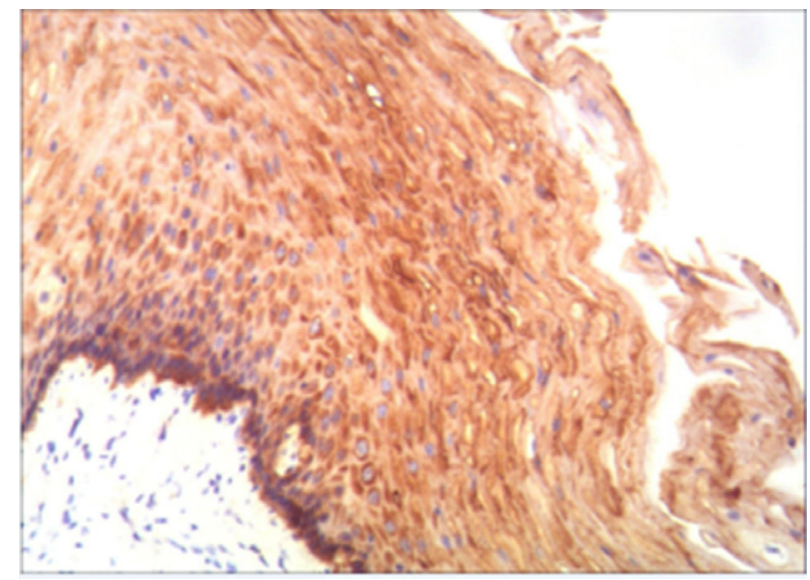

Figure 5. CADM1 protein in the form of brown granules in cytoplasm of normal esophagus tissues (+++, SP, 100X).

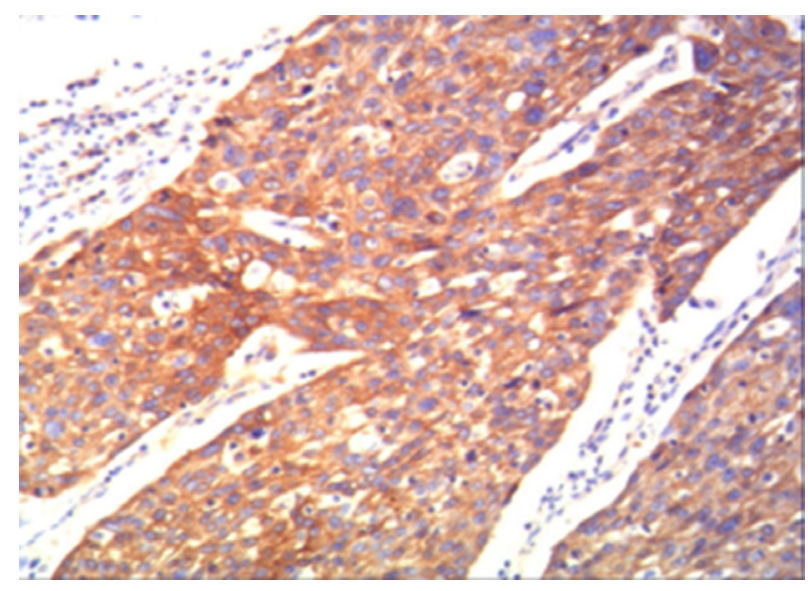

Figure 6. CADM1 protein in the form of brown granules in cytoplasm of esophageal carcinoma tissues (+++, SP, 100X).

Genetics and Molecular Research 16 (2): gmr16029178 


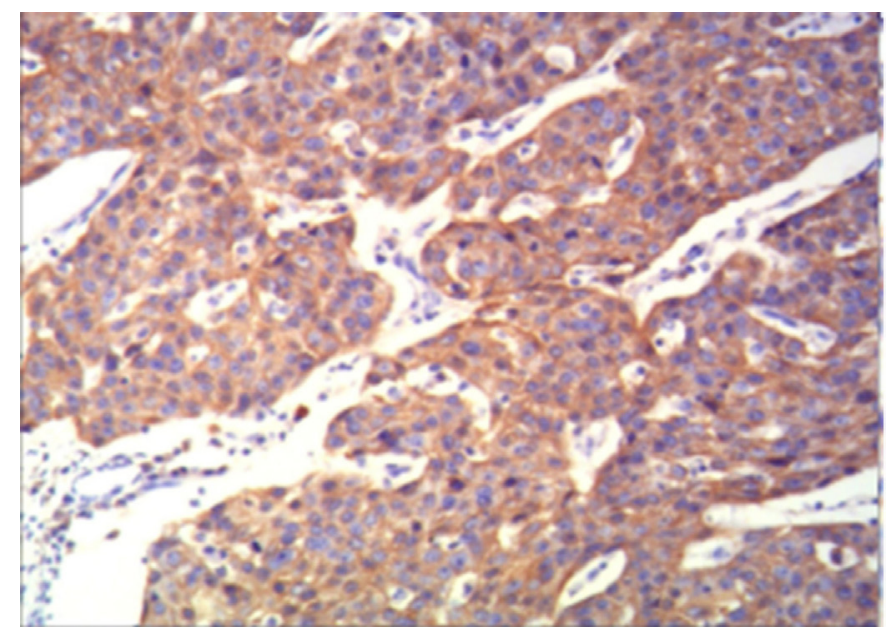

Figure 7. CADM1 protein in the form of yellow granules in cytoplasm of esophageal carcinoma tissues (+++, SP, 100X).

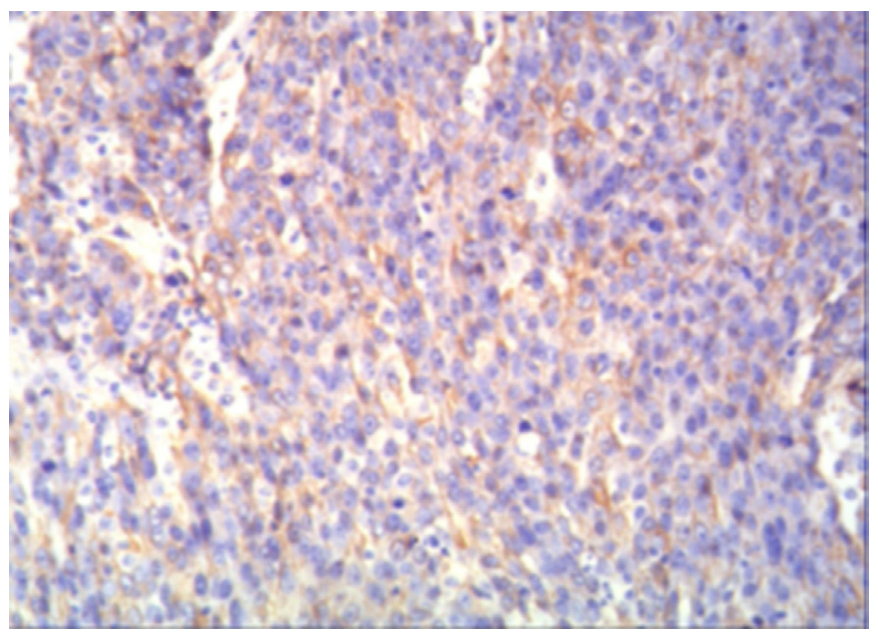

Figure 8. CADM1 protein in the form of pale yellow granules in cytoplasm of esophageal carcinoma tissues $(+++$, SP, 100X).

In 50 cases of ESCC, there were 20 cases (71.43\%) with positive expression of the CADM1 protein in 28 patients with non-lymph node metastasis, and there were only 7 cases $(31.82 \%)$ with positive expression of the CADM1 protein in 22 patients with lymph node metastasis. There was a significant difference in the positive expression rates of CADM1 protein between the two groups $\left(\chi^{2}=7.782, \mathrm{P}<0.01\right)$. The positive expression rate of CADM1 protein in patients in TNM stages I+II was significantly different from that of patients in stages III + IV $\left(\chi^{2}=9.522, \mathrm{P}<0.01\right)$. The positive expression rate of CADM1 protein in ESCC grades I, II, and III decreased, but there was no significant difference between the groups $(\mathrm{P}>0.05)$. There was no correlation between the positive expression rate of the CADM1 protein and gender, age, and other clinicopathological factors $(\mathrm{P}>0.05)$.

Genetics and Molecular Research 16 (2): gmr16029178 


\section{DISCUSSION}

CADM1 was identified by Kuramochi et al. in 2001 in their research on lung cancer. There are two transcriptosomes of 4.4 and $1.6 \mathrm{~kb}$ in CADM1 coding for the same protein. CADM1 is a transmembrane glycoprotein with 442 amino acids and comprises the extracellular, transmembrane, and cytoplasm domains. Most of the CADM1 expression is in the epithelial tissue; the extracellular domain of CADM1 protein has significant homology with other immunoglobulins. Therefore, we presumed that the CADM1 protein might participate in cell adhesion (Li and Zhang, 2006; Liang et al., 2011). According to van der Weyden et al. (2012), CADM1 expression was downregulated or repressed in epithelial tumor. Except for destroying cell adhesion, CADM1 might convey a signal to promote tumor metastasis and invasion. With the help of immunohistochemistry, Goto et al. (2005) detected lung adenocarcinoma in 93 cases and found that CADM1 expression levels were downregulated with the increase in the grade of malignancy. Takahashi et al. (2012) showed that abnormal CADM1 expression was related to breast cancer, especially the processes of invasion and metastasis. Surace et al. (2004) studied meningioma and showed that $48 \%$ of benign tumors in grade I, $69 \%$ of atypical meningiomas in grade II, and $85 \%$ of malignant meningiomas in grade III were related to the loss of CADM1 expression; the CADM1 expression levels were downregulated with increase in the grade of malignancy. These results showed that the loss of CADM1 gene expression was related to the decrease in survival rate of patients with meningioma.

In China, Yang et al. (2006) found that the expression rate of CADMI in invasive squamous carcinoma of the cervix was high. Ito et al. (2003) studied 36 cell lines and 56 tumor samples of human esophageal carcinomas, and found that CADM1 expression was downregulated in $75 \%$ of the cell lines and tissues. Additionally, in primary esophageal cancers, Lung et al. (2006) discovered that there was a higher incidence of loss of CADM1 expression in grade II, III, and IV cancers than in grade I.

These results showed that many tumors exhibit the loss of CADM1 expression, especially tumors with higher invasiveness. Therefore, CADM1 is a potential biomarker in the prognosis of the patients with different carcinomas after treatment. There were a few reports on the correlation of CADM1 mRNA expression with the prognosis of ESCC.

In our research study, we used RT-PCR and SP immunohistochemical method to detect and compare the levels of expression of CADM1 mRNA and protein in the ESCC tissues and adjacent tissues. The results showed that the expression of CADM1 mRNA and protein was different, but were higher in the normal tissues than in the tumor tissues of the patients with ESCC; this indicated that the CADM1 gene was linked with a mutation in ESCC. There was a correlation between the expression of CADM1 mRNA and protein and the pathogenesis and development of ESCC, which helped in providing a diagnosis and identifying ESCC with a reference value.

Additionally, we detected and compared the levels of expression of CADM1 mRNA and protein in ESCC samples with different TNM grades. The results showed that CADM1 mRNA expression levels were downregulated with increase in the grade of malignancy, but without a significant difference. However, levels of expression of CADM1 mRNA and protein in ESCC tissues with positive lymph node metastasis were significantly lower than that of those with negative lymph node metastasis. There was a significant difference in levels of CADM1 mRNA and protein expression between carcinoma samples of TNM grades I+II and III+IV, which indicated that loss of CADM1 expression was related to uncontrolled proliferation of

Genetics and Molecular Research 16 (2): gmr16029178 
cells in ESCC, and that the CADM1 gene might play a role in the invasion and metastasis of ESCC. In conclusion, the loss of CADM1 mRNA expression is a potential marker for the diagnosis of the metastasis and prognosis of ESCC.

In the later study, the relationship between the expression of CADM1 mRNA and protein and the prognosis of the patients was further investigated using a large amount of data and follow-up.

\section{Conflicts of interest}

The authors declare no conflict of interest.

\section{ACKNOWLEDGMENTS}

Research supported by the 2013 Nantong Social Science and Technology Innovation and Demonstration Projects (\#HS2013022).

\section{REFERENCES}

Goto A, Niki T, Chi-Pin L, Matsubara D, et al. (2005). Loss of TSLC1 expression in lung adenocarcinoma: relationships with histological subtypes, sex and prognostic significance. Cancer Sci. 96: 480-486. https://doi.org/10.1111/j.13497006.2005.00075.X

Ito T, Shimada Y, Hashimoto Y, Kaganoi J, et al. (2003). Involvement of TSLC1 in progression of esophageal squamous cell carcinoma. Cancer Res. 63: 6320-6326.

Kuramochi M, Fukuhara H, Nobukuni T, Kanbe T, et al. (2001). TSLC1 is a tumor-suppressor gene in human non-smallcell lung cancer. Nat. Genet. 27: 427-430. https://doi.org/10.1038/86934

Li DL and Zhang CJ (2006). Lung tumor suppressor gene 1: a new tumor suppressor gene. Shengmingdehuacue 26: 405.

Liang QL, Chen GQ, Li ZY and Wang BR (2011). Function and histopathology of a cell adhesion molecule TSLC1 in cancer. Cancer Invest. 29: 107-112. https://doi.org/10.3109/07357907.2010.543211

Lung HL, Cheung AK, Xie D, Cheng Y, et al. (2006). TSLC1 is a tumor suppressor gene associated with metastasis in nasopharyngeal carcinoma. Cancer Res. 66: 9385-9392. https://doi.org/10.1158/0008-5472.CAN-06-0590

Surace EI, Lusis E, Murakami Y, Scheithauer BW, et al. (2004). Loss of tumor suppressor in lung cancer-1 (TSLC1) expression in meningioma correlates with increased malignancy grade and reduced patient survival. J. Neuropathol. Exp. Neurol. 63: 1015-1027. https://doi.org/10.1093/jnen/63.10.1015

Takahashi Y, Iwai M, Kawai T, Arakawa A, et al. (2012). Aberrant expression of tumor suppressors CADM1 and 4.1B in invasive lesions of primary breast cancer. Breast Cancer 19: 242-252. https://doi.org/10.1007/s12282-011-0272-7

van der Weyden L, Arends MJ, Rust AG, Poulogiannis G, et al. (2012). Increased tumorigenesis associated with loss of the tumor suppressor gene Cadm1. Mol. Cancer 11: 29. https://doi.org/10.1186/1476-4598-11-29

Yang YX, Yang AH, Yang ZJ, Wang ZR, et al. (2006). Involvement of tumor suppressor in lung cancer 1 gene expression in cervical carcinogenesis. Int. J. Gynecol. Cancer 16: 1868-1872. https://doi.org/10.1111/j.1525-1438.2006.00656.x

Genetics and Molecular Research 16 (2): gmr16029178 